\title{
In-Depth Study of Coating Multimodal Porosity Using Ellipsometry Porosimetry in Desorption Scanning Mode Electronic Supporting Information
}

\section{Jérôme Loizillon'1, Bettina Baumgartner², Christophe Sinturel ${ }^{3}$, Marco Abbarchi ${ }^{1}$, Bernhard Lendl ${ }^{2}$, David Grosso ${ }^{1 *}$}

1 Institut Matériaux Microélectronique et Nanosciences de Provence, (IM2NP) - UMR CNRS 7334, AixMarseille Université, Faculté des Sciences de Saint Jérôme, 13397 Marseille Cedex 20, France

2 Research Division of Environmental Analytics, Process Analytics and Sensors, Institute of Chemical Technologies and Analytics, Technische Universität Wien, Getreidemarkt 9, 1060 Vienna, Austria

3 Interfaces, Confinement, Matériaux et Nanostructures (ICMN), UMR 7374 CNRS-Université d'Orléans, 1B Rue de la Férollerie, C.S. 40059, 45071 Orléans Cedex 2, France

david.grosso@univ-amu.fr

\section{Correlation Distances $\mathbf{d}_{\mathrm{z}}$}

All correlation distances $d_{z}$ were calculated using the following procedure. First, from each GI-SAXS pattern the distance $z$ between the beam center (intersection of the $Q_{y}$ and $Q_{z}$ axis) and the ellipse was measured in $\mathrm{mm}$ using fit2D software. Then, the diffraction angle $\theta$ can be obtained using the following relation :

$$
\theta=\frac{1}{2} \tan ^{-1}\left(\frac{Z}{L}\right)
$$

With $\mathrm{L}=1170 \mathrm{~mm}$ the sample-detector distance

The correlation distance $d_{z}$ was calculated using the Bragg equation :

$$
d_{z}=\frac{\lambda}{2 \sin \theta}
$$

With $\lambda=0.154 \mathrm{~nm}$ the wavelength used for the experiment.

\section{Wall Thicknesses t}

In other to deduce the wall thicknesses from the correlation distances $d_{z}$, it is necessary to consider a specific pore packing. In this case, since no well-defined diffraction spots were recorded on the GI-SAXS pattern (diffused continuous elliptical signals), we propose that the pore arrangement originates from a quasi-close pack organization of the micelles at very short scales (a few micelles), which is characterized by a correlation distance given by the GI-SAXS. Only the out-of-plane orientation is considered for simplicity. Due to the thermal contraction of the film, the initially spherical packed micelles are 
contracted along the $z$ axis, resulting in oblate spheroid pores (Figure S1). This contracted distance corresponds to the correlation distance $d_{z}$ obtained from the GI-SAXS pattern. The correlation distance before the contraction is equal to the correlation distance $d_{y}$ obtained from the SAXS pattern on the inplane axis. This is because $d_{y}$ is obtained from packing domains that were oriented with a $90^{\circ}$ rotation with respect to the domains represented on Figure $S 1$, meaning $d_{y}$ was oriented in the in-plane direction which is not affected by the thermal contraction of the film. This also means that the thermal contraction ratio is equal to the $d_{y} / d_{z}$ ratio, in this case 0.65 for every sample, in good accordance to what can be found in the literature for $\mathrm{F} 127^{1}$.
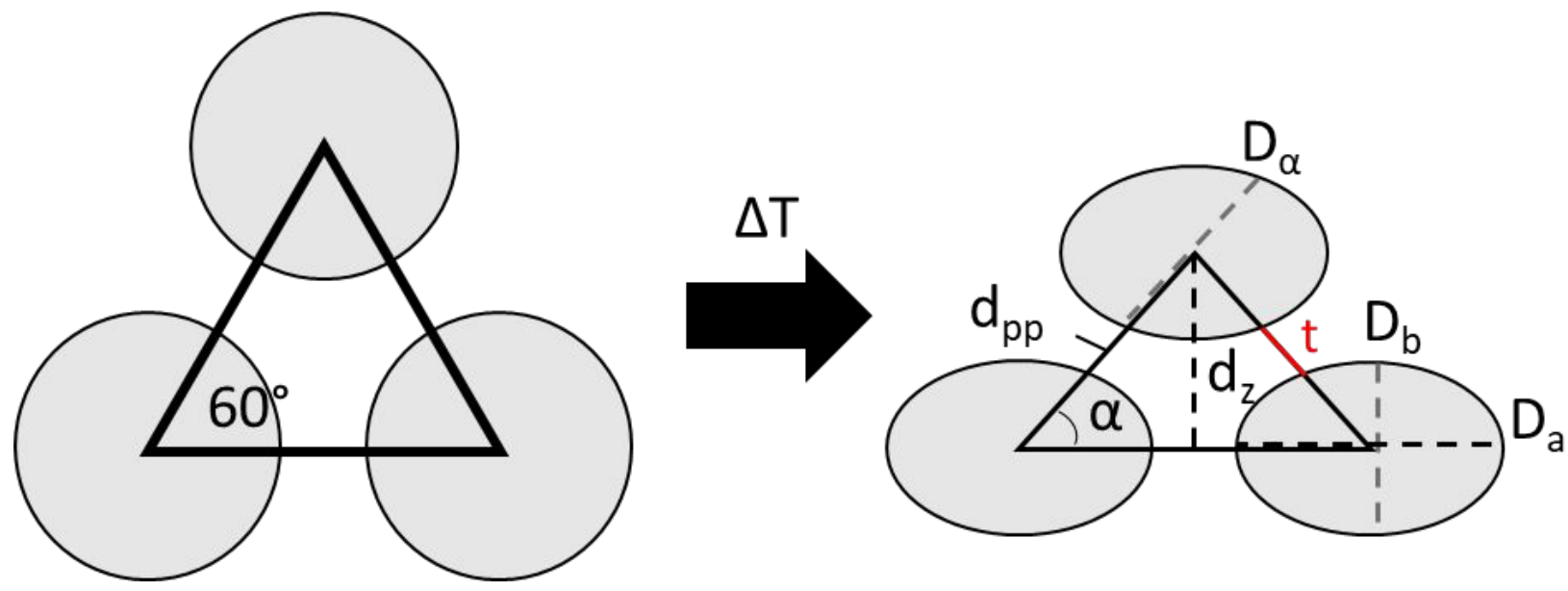

Figure S1: Thermal contraction of the initial spherical template micelles into oblate pores with $d_{z}$ the correlation distance, $d_{p p}$ the pore-to-pore distance, $t$ the wall thickness, $D_{a}, D_{b}$ and $D_{\alpha}$ the different diameters of the pores

Following this, $\alpha$, the angle after contraction can be obtained using the following equation:

$$
\propto=\tan ^{-1}\left(\frac{\tan 60}{\frac{d y}{d z}}\right)=48^{\circ}
$$

Then, the pore to pore distance $d_{p p}$ can be calculated using the following equation :

$$
d_{p p}=\frac{d_{z}}{\sin \alpha}
$$

$D_{\alpha}$ the pore diameter along the angle $\alpha$ is then calculated from the small and large diameters of the pores $D_{b}$ and $D_{a}$. $D_{b}$ is known from the pore size distribution obtained by ellipsometry porosimetry and $D_{a}$ is calculated from $D_{b}$ and the thermal contraction with the following relation :

$$
D_{a}=D_{b} \frac{d_{y}}{d_{z}}
$$

$D_{\alpha}$ can then be calculated using the polar equation of an ellipse : 


$$
D_{\alpha}=\frac{D_{a} D_{b}}{\sqrt{\frac{D_{a}^{2}}{4} \sin ^{2} \alpha+\frac{D_{b}^{2}}{4} \cos ^{2} \alpha}}
$$

At this point, the silica wall thickness $t$ between the pores is easily accessible by calculating the difference between the pore center to center distance $d_{p p}$ and the pore diameter $D_{\alpha}$ as in the following equation :

$$
t=d_{p p}-D_{\alpha}
$$

\section{REFERENCES}

(1) Boissiere, C.; Grosso, D.; Lepoutre, S.; Nicole, L.; Bruneau, A. B.; Sanchez, C. Porosity and Mechanical Properties of Mesoporous Thin Films Assessed by Environmental Ellipsometric Porosimetry. Langmuir 2005, 21 (26), 12362-12371. https://doi.org/10.1021/la050981z. 